\title{
Signal Filter Cut-off Frequency Determination to Enhance the Accuracy of Rail Track Irregularity Detection and Localization
}

\author{
Bhavana Bhardwaj, Raj Bridgelall, Leonard Chia, Pan Lu, Neeraj Dhingra
}

\begin{abstract}
A continuous condition monitoring system to detect and localize railroad track irregularities is achievable with inertial sensors onboard revenue service trains. However, the inaccurate geospatial position estimates of GPS receivers and the non-uniform sampling of inertial sensors adds noise and reduces signal strength. Consequently, the signal-to-noise ratio decreases, which leads to higher rates of false positives and false negatives. Appropriate signal filtering, alignment, and combination from multiple traversals can enhance the signal-to-noise ratio. However, it is not straightforward to determine the best cut-off frequency for the filter. This paper introduces a method that is suitable for any signal filtering approach. The frequency window of the resultant energy and variance of ensemble averaged FFTs informs the best cut-off frequency. The results affirm that a lowpass finite impulse response filter with the selected cutoff frequency progressively increases the signal-to-noise ratio with increasing filter order, thus demonstrating the effectiveness and practicality of the method.
\end{abstract}

Index Terms - Autonomous condition monitoring, digital signal filtering, non-destructive evaluation, sample rate.

\section{INTRODUCTION}

$\mathrm{T}$ HE railroad industry plays a crucial role in stimulating the economic growth of a nation. It transports a significant share of freight and moves millions of people every day. Regular maintenance of railroad assets is essential for their proper functioning and significantly improves ride quality, reduces track damage, extends maintenance intervals, and increases track life.

Deviations from the designed track geometry increase the risk of derailment by intensifying angular movements and linear accelerations [1]. Therefore, there is a need to monitor the condition of tracks continuously. However, the existing methods of measuring track geometry irregularities rely on manual inspections and heavily instrumented vehicles. This approach is laborious, relatively slow, expensive, and requires track closures or slow orders.

Advances in inertial sensors that integrate accelerometers, gyroscopes, and GPS receivers into a single device have led to their ubiquity and massive cost reduction [2].

B. Bhardwaj, R. Bridgelall, L. China, P. Lu, and N. Dhingra are with the Department of Transportation, Logistics, and Finance, North Dakota State University, ND 58108 USA. (Email: bhavana.bhardwaj@ ndsu.edu).
Hence, installing such sensors on revenue service trains can be affordable. Onboard sensors can transmit data to a centralized system that monitors automatically and continuously for inertial events produced by traversing irregular track geometry. Such a system can improve the cost-effectiveness of railroad resources by focusing their inspections on high-risk locations [3].

Smartphones currently have all the embedded sensor capabilities needed to develop and test the proposed condition monitoring system [4]. However, it is well known that the geospatial position estimates from low-cost GPS receivers are inaccurate mostly because of signal deterioration in urban environments and their low update rates [5]. The inertial sensors also suffer from bias instability [6]. Moreover, the non-uniform sample rate of the sensors results in additive signal noise and position alignment errors that reduce the signal-to-noise ratio (SNR) needed to detect and localize track irregularities reliably. In particular, false positives and false negatives can increase when SNR decreases.

The organization of this paper is as follows: Section II summaries related work. Section III describes the specifications and placement location of the sensors; also it provides the format and units of the GPS, accelerometer, and gyroscopic signal samples collected. Section IV develops the method for selecting the appropriate filter cut-off frequency. Section V evaluates the outcome of the method by comparing frequency windows of energy and variances of the ensemble average of the Fourier transforms. This section also demonstrates the effectiveness of the approach through SNR analysis. Section VI presents concluding remarks about the generalization and utility of the approach.

\section{LITERATURE REVIEW}

Vehicle vibrations and electromagnetic interference contribute to noise and other unwanted signal components in the inertial signal samples. Therefore, the selection of the appropriate signal filtering technique is necessary to employ a signal filter to minimize the noise and maximize the signal [6], [7]. Previous work attempted to address this problem by applying different signal filtering methods. Hasan et al. [7] proposed wavelet multi-resolution analysis to de-noise inertial measurement unit (IMU) signals by band-limiting the signal at the output of each inertial measurement sensor. Their results 
showed potential reductions of short-term errors (highfrequency noise) that provide more accurate position and velocity. Heirich et al. [8] analyzed the acceleration of the longitudinal, lateral, and vertical axis of the train in time and frequency domain. They used a low-cost IMU based on MEMS sensors to record the inertial data. A microelectromechanical system (MEMS) is a device in consumer electronics that can measure the acceleration of a moving train [9]. The low dynamics of the train motion allow authors of reference [8] to use a low-pass filtering approach to improve the signal-to-noise ratio (SNR). They first performed a fast Fourier transform (FFT) and then applied a finite impulse response (FIR) low-pass filter with a window of 100 samples and a cutoff frequency of $2 \mathrm{~Hz}$. Real et al. [10] introduced a procedure for obtaining the vertical rail profile by using inertial methods. They modeled the interaction between the train and the track as a system of springs and dampers with two degrees of freedom. They then used an FFT to transform the signals into the frequency domain. Samé et al. [11] applied a regression model to reduce asymmetrical noise from signals obtained from a railway diagnostic application. Another study [12] adopted a signal processing point of view to diagnose rail corrugation. They applied a model-based time-frequency tool to introduce additional information about the spectral content of the signal. Jian et al. [13] used a tracking filter to improve the SNR of a Doppler signal.

In general, these methods can be categorized as standard digital filters that include IIR filter, FIR filter, and notch filter. Prior knowledge of the frequency response parameter is required to design such filters. However, in the absence of such information, it is not possible to design standard filters because of the changing nature of the filter's requirements [14]. Thus, the inability of such a system to work with limited information makes it less suitable for general application [15]. Consequently, in such a situation, an adaptive filtering approach is favored. For instance, authors in [16] employed a noise-canceling technique by using adaptive filtering to improve SNR. Similarly, Nagal et al. [17] implemented an adaptive filtering approach in the time and frequency domains. The results show that the SNR of the output signal rises about 8-9 times more in the frequency domain than the time domain. Alam et al. [6] proposed an adaptive data pre-processing technique by using variable bandwidth filtering. Another related area of adaptive filtering research focused on the estimation of train longitudinal velocity based on locomotive wheelset velocity [18],[19]. However, adaptive filtering also has some shortcomings, which the previous studies did not highlight. The adaptive process requires the use of a more complex cost function [20] and bank of filters to evaluate the SNR in a closed-loop manner. Hence, convergence can be slow and require high computational complexity. Adaptive filters are not guaranteed to be numerically stable, which limits their use in practice [21]. Adaptive filtering operates on a single signal stream and does not guarantee convergence to a local minimum that would maximize the SNR.
To overcome the limitations of adaptive filtering, we contributed a method to inform the cutoff frequency selection for a FIR low-pass filter that maximizes the SNR, with low computational complexity and high stability for practical use. The method achieves this by combining the FFTs from multiple signal streams of the same traversal to produce an ensemble average FFT (EA-FFT). Some heuristics based on observations of the global EA-FFT pattern validates the cutoff frequency selection.

\section{INERTIAL SENSOR DATA}

Due to non-disclosure agreements with the railroads, this paper simulated data from railroad track traversals by driving a paved road segment (Cell 40) of the Minnesota road (MnROAD) research facility in the United States. A smartphone application (app) named pavement analysis via vehicle electronic telemetry (PAVVET) logged the inertial and geospatial data from a sedan [22].

\section{A. Sensor Specification and Setup}

This study uses sensors embedded in the Apple iPhone 4s. They include a global positioning system (GPS) receiver [23], an STMicroelectronics LIS $_{331} \mathrm{DLH}$ accelerometer, and a $\mathrm{L}_{3} \mathrm{G}_{4} 200 \mathrm{D}$ gyroscope [24]. The GPS receiver sampled at a nominal rate of one $\mathrm{Hz}$. The accelerometer sampled at a nominal rate of $128 \mathrm{~Hz}$. The smartphone was mounted flat onto the dashboard with the $y$-axis pointing in the direction of travel [23].

\section{B. Data Format}

Table I shows the format of a fragment of the data collected from the smartphone-embedded inertial and GPS sensors. The time variable is in milliseconds from the start of the data collection. The GPS data is latitude (Lat) and longitude (Lon) in decimal format. The ground speed (GSpeed) is in units of $\mathrm{m} \mathrm{s}^{-1}$. The g-force values of Gx, Gy, and $\mathrm{Gz}$ are accelerations in the lateral, longitudinal, and vertical directions, respectively. The integrated gyroscope provides the pitch, roll, and yaw angles of the sensor orientation in degrees. The RotX, RotY, and RotZ signals are the angular rotations in degrees-persecond around the $\mathrm{X}, \mathrm{Y}$, and $\mathrm{Z}$ axis, respectively.

The data consists of 53 traversals at nearly uniform speeds. The authors verified that the inertial signal patterns observed from traversing road bumps are similar to those observed when a train traverses track irregularities. The Cell 40 dataset contains a consistent inertial event from a narrow bump at an asphalt-concrete joint at the beginning of each traversal. The distinct inertial signal from this bump produced a first major valley (FMV) in the accelerometer signal. Hence, the FMV served as the known ground truth for comparison with the GPS positions reported for that irregularity. Therefore, the FMV provided a means to quantify the accuracy of four heuristic alignment algorithms proposed in a previous study [25]. Each alignment algorithm extracted signals from approximately 
equal length traversals within two geofences along the roadway. The algorithms produced different results as characterized by the distribution of the reported positions of the FMV, and the distribution of the extracted segment lengths. This analysis selected the best algorithm of the four to align the segments before truncation to produce signals from approximately equal length segments.

The non-uniform sampling of the accelerometer caused the sample period to vary among traversals. Furthermore, the smartphones sampled at different mean sample rates, despite setting the sample rate request to a constant value of $128 \mathrm{~Hz}$. Fig. 1 shows the distribution of the sample period aggregated from the accelerometer data across all traversals. Simulations confirmed that non-linear sampling adds noise and decreases signal strength. Therefore, a signal filter is necessary to counter the reduction in $\mathrm{SNR}$, and to preserve the quality of the signals that represent inertial events.

\section{METHOD}

The individual FFTs of the inertial signals from each traversal produce patterns in the spectral domain that do not clearly distinguish between information and noise. The EA-FFT enhances the clarity of the patterns by canceling randomness and boosting the correlated portions of the patterns. Subsequently, the sharpened pattern revealed a clearer breakpoint between information and noise. Fig. 2 summarizes the individual processes of the overall method. Fig. 3 illustrates the workflow to determine the filter cut-off frequency.

After obtaining the signals from approximately equal length traversals, the remaining procedure was to 1) compute the EA-FFT from the individual FFTs of the inertial signals, 2) compute energy and variance windows along the frequency range, 3) determine the appropriate cut-off frequency, and (4) apply a low-pass digital filter to each inertial signal. Each FFT used the mean sampling frequency (Fs) of its individual signal. However, the mean sample frequency varies among traversals. Therefore, the EA-FFT algorithm aligned the frequency bins before averaging the non-zero magnitudes [26] . A frequency bin size of $1 \mathrm{~Hz}$ provided sufficient resolution to compute $5 \mathrm{~Hz}$ energy and variance windows for cut-off frequency selection. In particular, the observed transition of energy and variance from high to consistently lower values pointed to the appropriate cut-off frequency for a low-pass noise filter.

\section{A. FFT and Ensemble Average FFT}

The Fast Fourier transform (FFT) [27] is an efficient method of computing the discrete Fourier transform (DFT) $X_{j}$ of $x_{l}$ as follows:

$$
\begin{aligned}
& X_{j}=\sum_{l=0}^{N-1} x_{l} W^{-j l} \quad W=\exp \frac{2 \pi \sqrt{-1}}{N}, \\
& \mathrm{j}=0, \ldots, \mathrm{N}-1
\end{aligned}
$$

where each $x_{l}$ is an equally spaced sample of a function $x(t)$, $l$ represents the time index, and $j$ represents the frequency index. $\mathrm{N}$ is an integral power of 2.
The EA-FFT is the mean value of all spectral magnitudes within a frequency bin $x$ such that

$$
X_{N x}=\frac{1}{N} \sum_{i=1}^{N} X_{i x}
$$

where $X_{N x}$ is the ensemble average of $N$ FFTs across bin $x$, and $X_{i x}$ is the magnitude of the FFT of an individual signal within frequency bin $x$. Fig. 4 shows the EA-FFT of the signals extracted from the approximately equal length segments after position alignment. Fig. 4 also illustrates the differences between the FFT of two individual signals, and the EA-FFT.

\section{B. Energy}

Signal processing [28] defines the energy of a finite signal $\mathrm{x}(\mathrm{n})$ as

$$
\mathrm{E}_{\mathrm{s}}=\sum_{\mathrm{n}=0}^{\mathrm{N}-1}|\mathrm{x}(\mathrm{n})|^{2}
$$

where Es is the energy of a signal, $\mathrm{n}$ is the sample number, and $N$ is the total number of samples. Fig. 5a shows the energy of the EA-FFT along $5 \mathrm{~Hz}$ windows.

\section{Variance}

Signal variance indicates how much a signal varies about the average value [29]. The variance of a signal is

$$
\sigma^{2}=\frac{1}{N-1} \sum_{\mathrm{i}=0}^{\mathrm{N}-1}\left[\mathrm{x}_{\mathrm{i}}-\mu\right]^{2}
$$

where the signal samples are $\mathrm{x}_{\mathrm{i}}, \mu$ is the signal mean, $N$ is the number of samples and $\sigma^{2}$ is the signal variance [29]. Fig. 5b shows the variance of the EA-FFT along $5 \mathrm{~Hz}$ windows. The observation is that for both the variance and the energy, there is an observable transition at $25 \mathrm{~Hz}$ between signal and, probable noise.

\section{Finite Impulse Response (FIR) Low-Pass Filtering}

The definition of a low-pass FIR filter of order $\mathrm{N}$ is [30]:

$$
\begin{aligned}
& y[n]=b_{0} x[n]+b_{1} x[n-1]+\cdots+b_{N} x[n-N] \\
& \text { or }
\end{aligned}
$$$$
y[n]=\sum_{i=0}^{N} b_{i} x[n-i]
$$

where $x[n]$ is the input signal, $y[n]$ is the output signal, and $\mathrm{N}$ is the filter order. Each coefficient $b_{i}$ is the value of the impulse response at the $\mathrm{i}^{\text {th }}$ instant for $0 \leq i \leq N$. The impulse response of the filter is:

$$
h[n]=\sum_{i=0}^{N} b_{i} \delta[n-i]=\left\{\begin{array}{cc}
b_{n} & 0 \leq \mathrm{n} \leq \mathrm{N} \\
0 & \text { otherwise }
\end{array}\right.
$$

Fig. 5c compares the unfiltered (black) and filtered (gray) signals. The delay in the filtered signal is due to the phase response of the FIR filter. It does not present an issue in the subsequent analysis because the filter will delay all signals similarly, and the algorithms can estimate and correct the distance offset accordingly. All the mathematical expressions can be implemented in any software such as python, C, SAS, Matlab and other software applications.

\section{EXPERIMENT ANALYSIS AND RESULT}

The SNR analysis demonstrates the effectiveness of the proposed method by comparing the filtered and unfiltered 
signal to validate improvements in SNR. The results show that the method of EA-FFT with statistical decision criteria is effective for informing a cut-off frequency to digitally low-pass filter the inertial signals. From Fig. 4, it is evident that the windowed ensemble averaging provides a clearer picture of the frequency transition from signal to noise. Also, it shows that Ensemble Average (EA) reduces noise and enhances correlated energy features in the spectral representation of the signal. Subsequently, there is agreement among the EA-FFTs on the frequency at which both the energy and variance in the spectral windows reach a minimum before rising again slightly. In particular, both the energy and variance windows of the EA-FFT shows such a transition at $25 \mathrm{~Hz}$.

\section{A. Filtered Signal vs. Unfiltered Signal}

Fig. 5c shows that the filter effectively removed noise while preserving the quality and strength of the inertial signature. The procedure can use any low-pass filter, including infinite impulse response (IIR) filters and is independent of the data collection apparatus and their operating systems.

\section{B. SNR Improvement Validation}

The filter order determines the degree to which the filter attenuates noise. A poor choice of cut-off frequency could result in the attenuation of both signal and noise, and subsequently no improvement in SNR. Therefore, the observation of SNR improvements with increasing filter order validates the cut-off frequency selection because the filter reduces noise while preserving the strength of the desired signal.

Fig. 5d shows that after applying a low-pass FIR filter with the selected cut-off frequency, the SNR steadily increased with the order of the filter. The SNR shown at order zero is for the unfiltered signal. This procedure prepared the inertial signals for the remaining stages of an application that involves signal position realignment, distance interpolation, and truncation to obtain signals from approximately equal length traversals for feature extraction and feature ensemble averaging.

\section{CONCLUSION}

Resolving issues such as non-uniform sample rate that adds noise and decreases the signal strength enables the placement of sensors in vehicles to monitor road or railroad condition. This work showed that appropriate signal filtering and alignment for ensemble averaging could be effective in improving the SNR for subsequent feature extraction. However, an objective determination of the filter cut-off frequency is necessary. From the individual FFTs, it is difficult to see a clear pattern that separates signal from noise. Hence, this paper contributes a method that ensemble averages the individual FFTs (EA-FFT) from the approximately equal length and position aligned inertial signals to enhance the clarity of the underlying pattern. The subsequent application of two statistical methods to frequency windows of the EA-FFT provides an objective means for selecting a frequency threshold where signal transitions to noise.

This proposed EA-FFT method to identify the cut-off frequency of a low-pass noise filter can be generalized to any sensor data that meet at least two criteria. The first is that the signal contains both signal and noise. The second is that the data source is from multiple traversals of a segment of some transportation network such as a rail track or a roadway. Such data produces distinct inertial signal patterns or features in common time windows. Moreover, variable train speed, GPS position registration errors, relatively slow update rates of the GPS receivers, and the uneven sampling rate of their inertial sensors result in feature alignment errors that can degrade the signal-to-noise ratio. Therefore, it is necessary to align and equalize the position of similar features or responses across multiple traversals.

Future work will focus on developing the methods of feature extraction, feature enhancement, and modeling to enhance the detection and localization accuracy of railroad track irregularities.

\section{ACKNOWLEDGMENT}

The work presented in this paper was conducted with support from North Dakota State University and the Mountain-Plains Consortium, a University Transportation Center funded by the U.S. Department of Transportation. The contents of this paper reflect the views of the authors, who are responsible for the facts and accuracy of the information presented.

\section{REFERENCES}

[1] X. Zhang, L. Jia, X. Wei, and N. Ru, "Railway track condition monitoring based on acceleration measurements," in Proceedings of the 2015 27th Chinese Control and Decision Conference, CCDC 2015, Qingdao, China, 2015, pp. 923-928.

[2] R. W. Ngigi, C. Pislaru, A. Ball, and F. Gu, "Modern techniques for condition monitoring of railway vehicle dynamics," J. Phys. Conf. Ser., vol. 364, no. 1, pp. 0-12, 2012.

[3] R. Schenkendorf and B. Dutschk, "Improved Railway Track Irregularities Classification by a Model Inversion Approach," Third Eur. Conf. Progn. Heal. Manag. Soc. 2016, Bibao, Spain, 2016, pp. 358-365, 2016.

[4] N. D. Lane, E. Miluzzo, H. Lu, D. Peebles, T. Choudhury, and A. T. Campbell, "A survey of mobile phone sensing," IEEE Communications Magazine, vol. 48, no. 9, pp. 140-150, 2010.

[5] N. A. Ali and M. Abu-Elkheir, "Improving localization accuracy: Successive measurements error modeling," Sensors (Switzerland), vol. 15, no. 7, pp. 15540-15561, 2015.

[6] M. Alam and J. Rohac, "Adaptive data filtering of inertial sensors with variable bandwidth," Sensors (Switzerland), vol. 15, no. 2, pp. 3282-3298, 2015.

[7] A. M. Hasan, K. Samsudm, A. R. Ramli, and R. S. Azmir, "Waveletbased pre-filtering for low cost inertial sensors," Journal of Applied Sciences, vol. 10, no. 19. pp. 2217-2230, 2010.

[8] O. Heirich, A. Lehner, P. Robertson, and T. Strang, "Measurement and analysis of train motion and railway track characteristics with inertial sensors," in 14th Internaltional IEEE Conference on Intelligent Transportation Systems, Proceedings, ITSC, Washington, DC, USA, 2011, pp. 1995-2000.

[9] D. Milne et al., "Proving MEMS Technologies for Smarter Railway Infrastructure," in The 3rd Internal Conference on Transportation Geotechnics (ICTG 2016), 2016, vol. 143, pp. 1077-1084.

[10] J. Real, P. Salvador, L. Montalbán, and M. Bueno, "Determination of rail vertical profile through inertial methods," Proc. Inst. Mech. 
Eng. Part F J. Rail Rapid Transit, vol. 225, no. 1, pp. 14-23, 2011.

[11] A. Samé, L. Oukhellou, E. Côme, and P. Aknin, "Mixture-modelbased signal denoising," Adv. Data Anal. Classif., vol. 1, no. 1, pp. 39-51, 2007.

[12] C. Hory, L. Bouillaut, P. Aknin, and B. Verte, "Monitoring rail corrugation in the mileage-wavelength domain using the ARCAP method," in 10th International Conference on Railway Engineering RE, 2009, vol. 9 .

[13] Z. Jian and L. Xingwu, "Research on laser Doppler velocimeter for vehicle self-contained inertial navigation system," Opt. Laser Technol., vol. 42, no. 3, pp. 477-483, 2010.

[14] F. Ali, P. Rawat, and S. Malvia, "Comparative Analysis and Survey of LMS and RLS Adaptive Algorithms," Int. J. Comput. Appl., vol. 161, no. 3, pp. 26-29, 2017.

[15] E. A. Oni, I. D. Olatunde, and K. O. Babatunde, "Improvement of Audio Signal Quality Using Adaptive Filtering and It ' $\mathrm{s}$ Performance Advantage over Non - Adaptive ( Linear ) Filtering," Intern. J. Electr. Electron. Sci., vol. 5, no. 2, pp. 39-45, 2018.

[16] B. Liang, S. Iwnicki, A. Ball, and A. E. Young, "Adaptive noise cancelling and time-frequency techniques for rail surface defect detection," Mech. Syst. Signal Process., vol. 54, pp. 41-51, 2015.

[17] R. Nagal, P. Kumar, and P. Bansal, "An Approach to Implement LMS and NLMS Adaptive Noise Cancellation Algorithm in Frequency domain," in In 2014 5th intrnational ConferenceConfluence The Next Generation Information Technology Summit (Confluence), Noida, India, 2014, pp. 871-876.

[18] P. Pichlík and J. Zděnek, "Train velocity estimation method based on an adaptive filter with fuzzy logic," J. Electr. Eng., vol. 68, no. 2, pp. 125-131, 2017.

[19] Fangjun Jiang and Zhiqiang Gao, "An adaptive nonlinear filter approach to the vehicle velocity estimation for ABS," in Proceeding ofthe 2000 IEEE International Conference on Control Applications, Anchorage, Alaska, USA, 2000, vol. 1, pp. 490-495.

[20] S. Garg and R. Sood, "A Literature Survey based on Adaptive

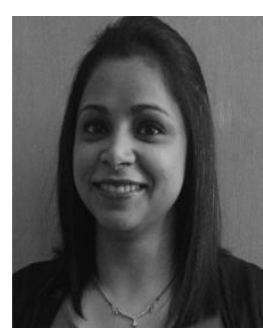

Bhavana Bhardwaj is working toward her Ph.D. degree within the Department of Computer Science and is a research assistant in the Department of Transportation, Logistic, and Finance at North Dakota State University. Her research interests include transportation safety, data mining techniques in transportation, sensor application in transportation and their application to signal processing.

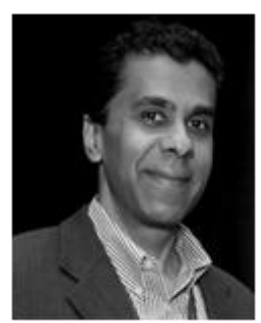

Dr. Raj Bridgelall (M'91-SM'02) Bridgelall received a $\mathrm{Ph} . \mathrm{D}$. degree in transportation and logistics at the North Dakota State University (NDSU), Fargo in 2015. He is presently program director of the $\quad$ SMARTSe ${ }^{\text {SM }} \quad$ Intelligent Transportation Systems Center, and assistant professor of Transportation.
Algorithms," Res. Cell An Int. J. Eng. Sci., vol. 17, no. 1, pp. 217221,2016

[21] S. Zhao, "Performance Analysis and Enhancements of Adaptive Algorithms and Their Applications," Ph.D. dissertation, School of Computer Engineering, Nanyang Technological University, 2009.

[22] R. Bridgelall, Y. Huang, Z. Zhang, and F. Deng, "Precision enhancement of pavement roughness localization with connected vehicles," Meas. Sci. Technol., vol. 27, no. 2, 2016.

[23] R. Bridgelall, "Inertial Sensor Sample Rate Selection for Ride Quality Measures," J. Infrastruct. Syst. ASCE, vol. 21, no. 2, p. 04014039, 2014.

[24] A. Allan, "Using the Accelerometer," in Basic Sensors in iOS, 1st ed. CA, USA: O'Reilly Media, Inc., 2011, pp. 37-53.

[25] N. Chia, Leonard; Bhardwaj, Bhavana; Bridgelall, Raj; Lu, pan; Tolliver, Denver; Dhingra, "Transportation Research Record Heuristic Methods of Inertial Signal Alignment to Detect and Locate Railtrack," in Presented at the 98th Annual Meeting of the Transportation Research, Washington, DC, USA, 2019.

[26] W. K. George, P. D. Beuther, and J. L. Lumley, "Processing of Random Signals," in Proceeding of the Dynamic Flow Conference 1978 on Dynamic Measurements in Unstready Flows, Springer, Dordrecht, 1978, pp. 757-758.

[27] J. W. Cooley and J. W. Tukey, "An Algorithm for the Machine Calculation of Complex Fourier Series," Math. Comput., vol. 19, no. 90, p. 297, 2006.

[28] L. F. Chaparro, "Discrete-Time Signals and Systems," in Signals and Systems using MATLAB, 3rd ed., Elsevier, 2011, pp. 451-509.

[29] S. W. Smith, "Statistics, Probability and Noise", in The Scientist and Engineer's Guide to Digital Signal Processing, 1st ed. San Diego, CA, USA: California Technical Publishing, 1997, pp. 11-34.

[30] E. Punskaya, "Design of FIR Filters," [Online]. Available: www. sigproc. eng. cam. ac. uk/ op205., 2005.

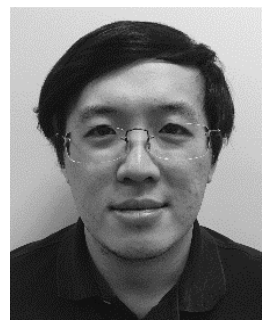

Leonard Chia is currently working toward his Ph.D. degree with the Department of Transportation, Logistics, and Finance at North Dakota State University. His research interests include asset management, sensor application in infrastructure, GIS application in transportation, and transportation safety.

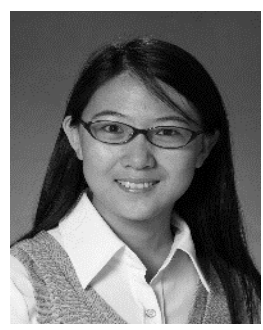

Dr. Pan Lu became a member of IEEE in 2017. She received a Ph.D. degree in transportation and logistics from North Dakota State University in 2011. Since 2017, Dr. Lu has been an associate professor in the College of Business at NDSU.

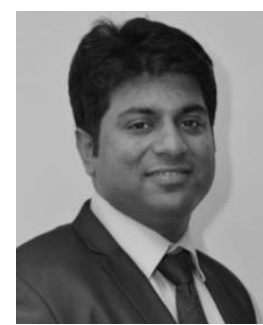

Neeraj Dhingra is currently working toward a Ph.D. degree within the Department of Transportation, Logistics, and Finance at North Dakota State University. His research interests include data mining techniques in transportation, transportation safety, GIS application in transportation and railway safety, and project management. 
TABLE I

Format of Data Sample

\begin{tabular}{ccccccccccccc} 
Time & Gz & Lat & Lon & GSpeed & Pitch & Roll & Yaw & Gx & Gy & RotX & RotY & RotZ \\
\hline 44.142 & -1.057 & 45.263 & -93.71 & 9.586 & 6.693 & 4.886 & -0.319 & -0.088 & -0.152 & 3.177 & 0.629 & -0.452 \\
46.768 & -1.216 & 45.263 & -93.71 & 9.586 & 6.693 & 4.886 & -0.319 & 0.047 & -0.241 & 3.177 & 0.629 & -0.452 \\
50.26 & -1.087 & 45.263 & -93.71 & 9.586 & 6.693 & 4.886 & -0.319 & 0.026 & -0.272 & 3.177 & 0.629 & -0.452 \\
62.927 & -0.854 & 45.263 & -93.71 & 9.586 & 6.741 & 4.903 & -0.329 & -0.002 & -0.212 & 1.246 & -0.13 & -0.332 \\
73.909 & -0.912 & 45.263 & -93.71 & 9.586 & 6.752 & 4.907 & -0.332 & 0.022 & -0.161 & 1.865 & 0.214 & -0.258 \\
86.754 & -0.942 & 45.263 & -93.71 & 9.586 & 6.776 & 4.908 & -0.341 & 0.038 & -0.144 & 2.005 & -0.67 & -0.189 \\
\hline
\end{tabular}

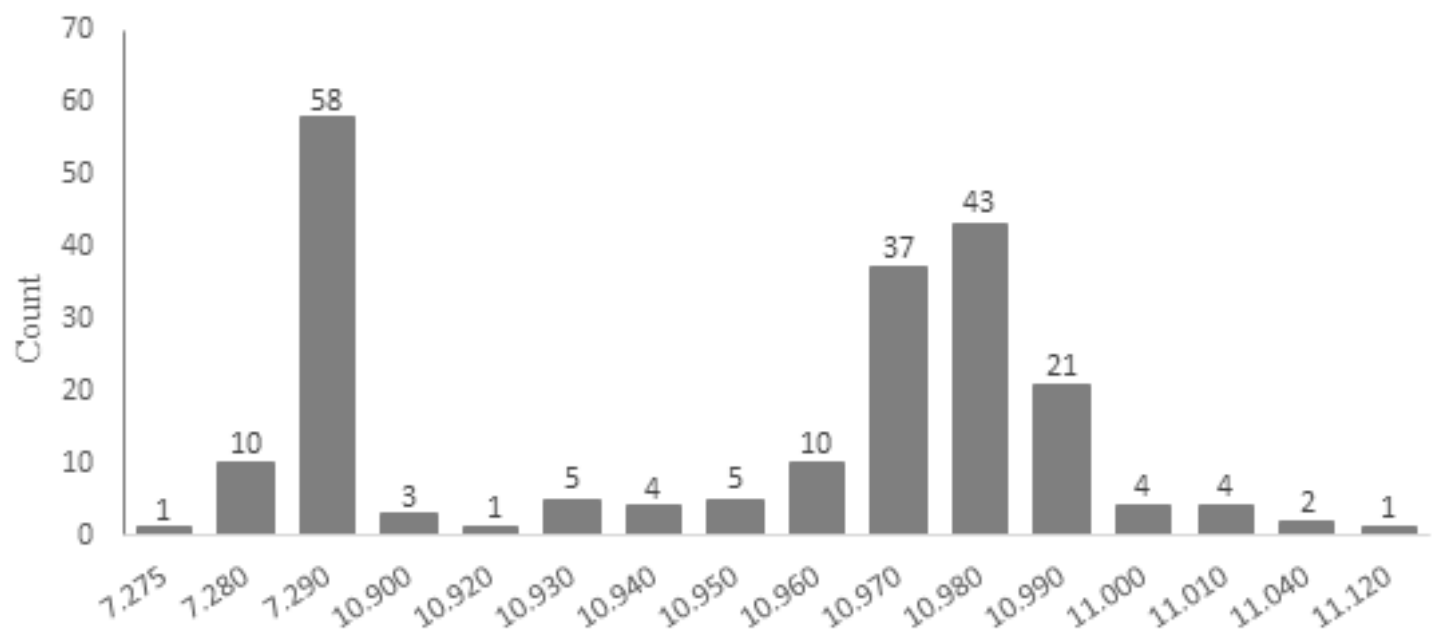

Sample Period (Milliseconds)

Fig. 1. Distribution of sample periods within and across devices.

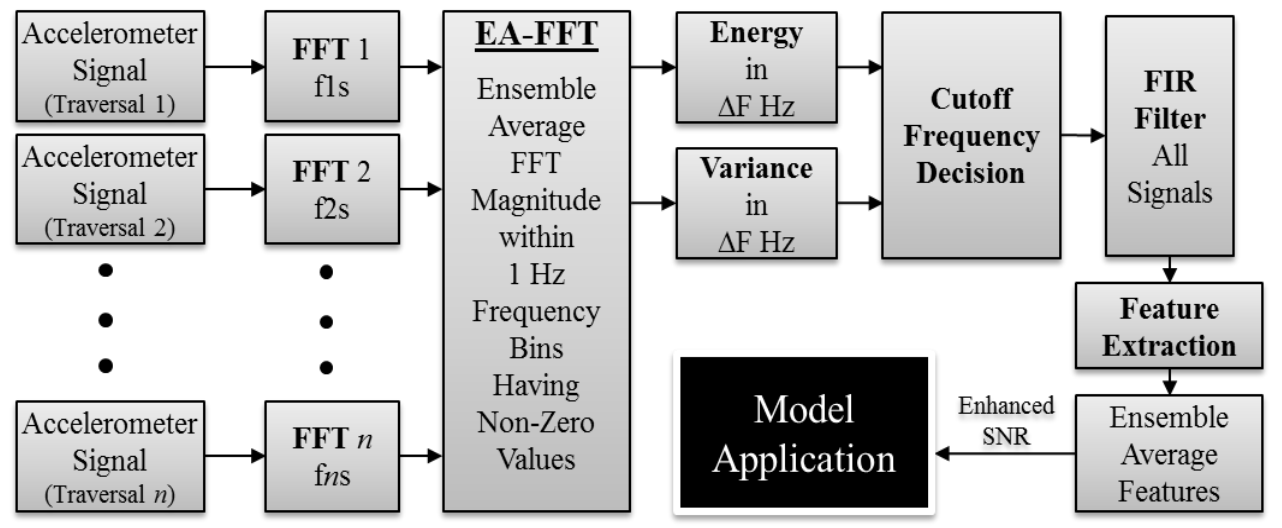

Fig. 2. Method of determining the signal filter cut-off frequency in preparation for feature extraction and their application. 


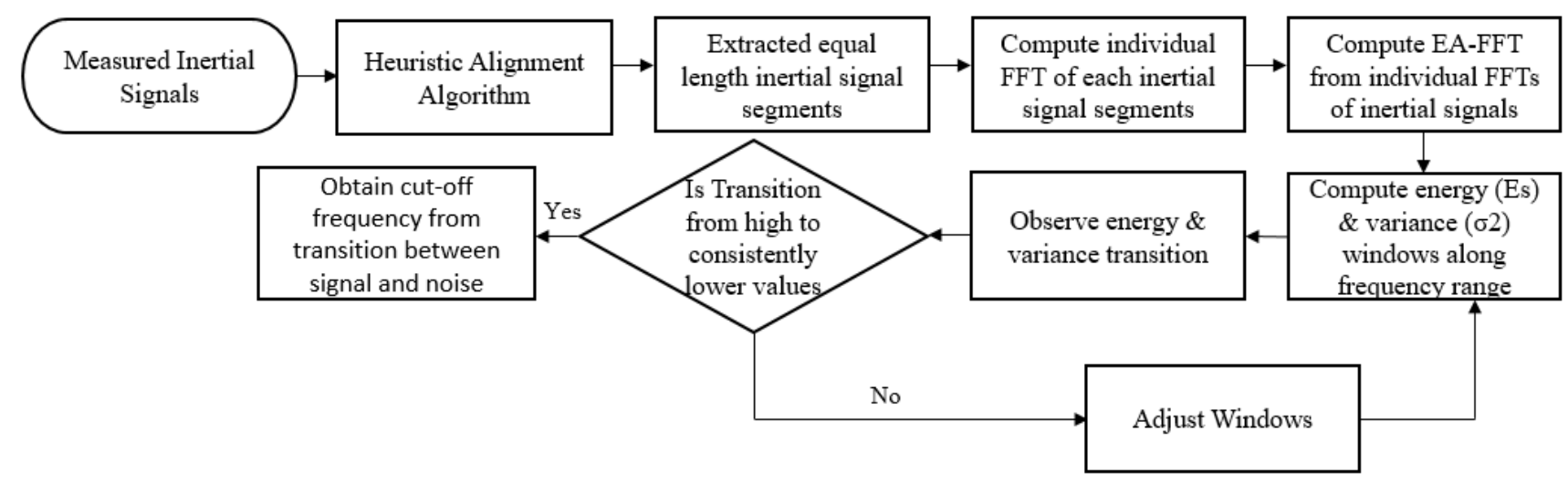

Fig.3. Workflow to determine the sensor signal cut-off frequency.

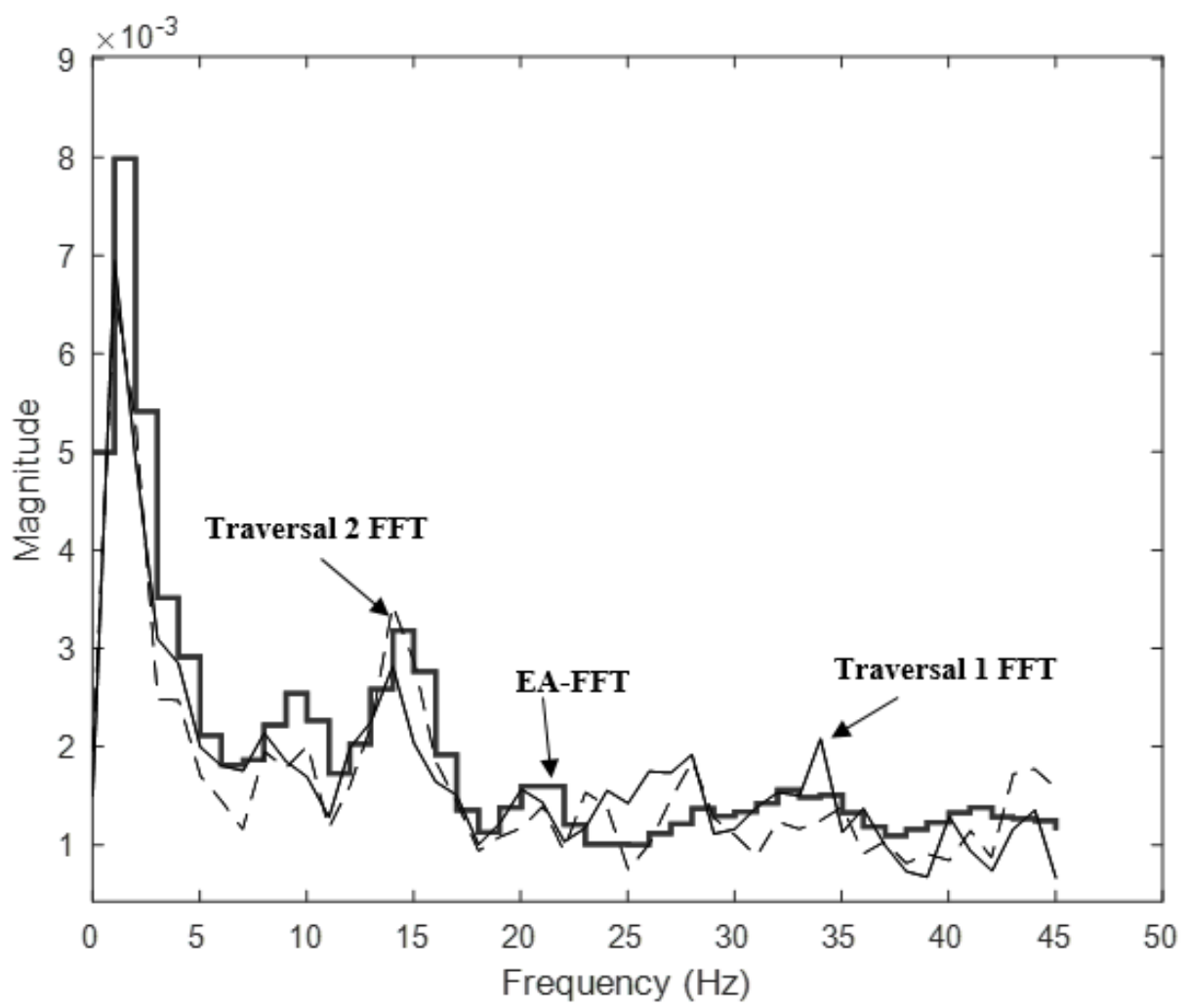

Fig. 4. The difference between the FFT of individual signals and the EA-FFT illustrates the improvement in pattern clarity. 
a)

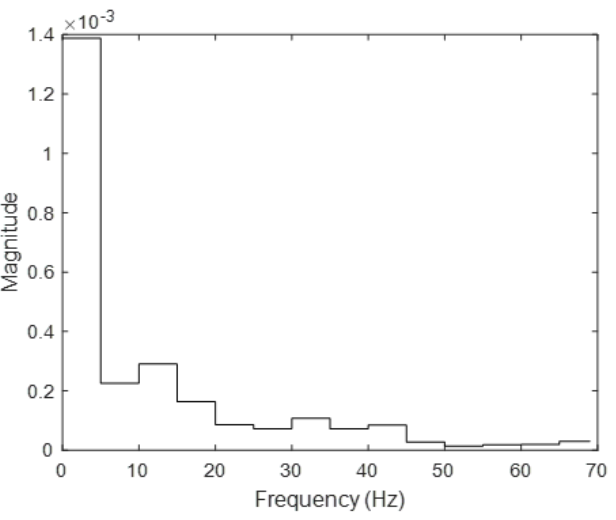

c)

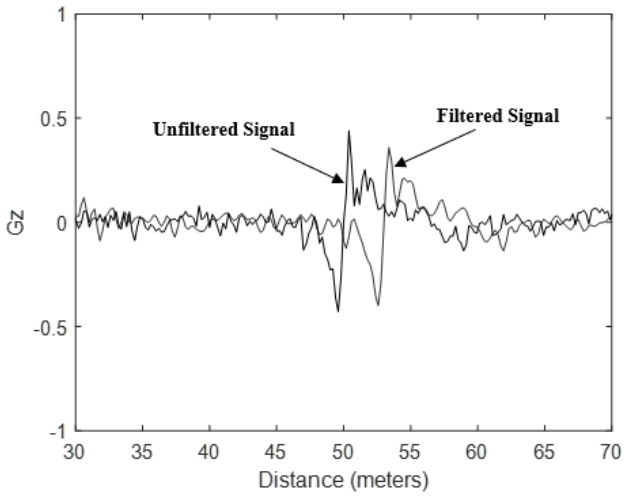

b)

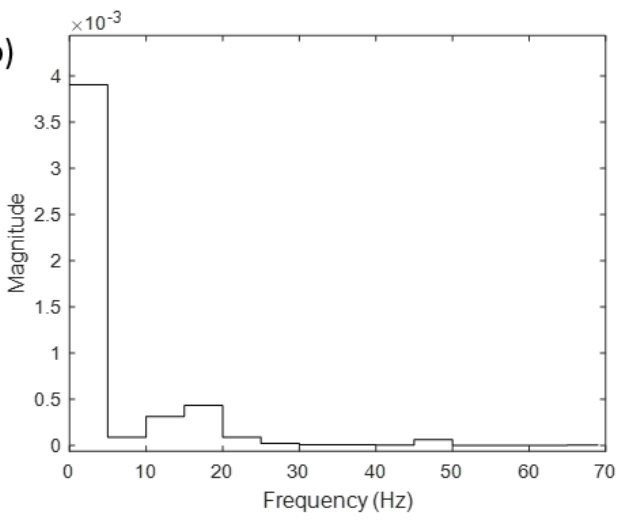

d)

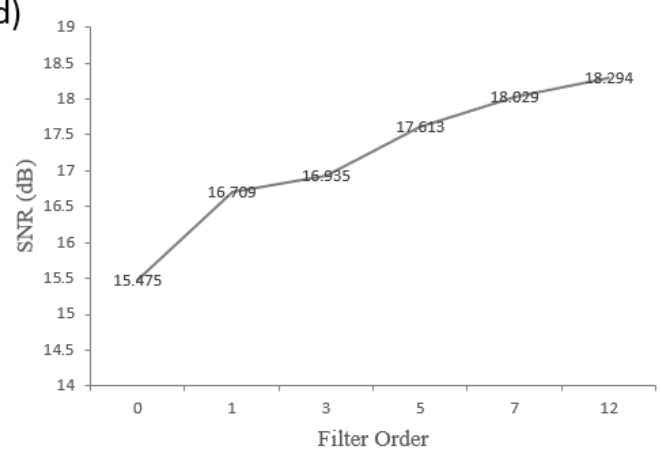

Fig 5. a) Energy windows of the EA-FFT, b) variance windows of the EA-FFT, c) the unfiltered and filtered signals, and d) the SNR as a function of filter order (zero order is the unfiltered signal) demonstrates the effectiveness of the cutoff frequency selection. 